\title{
Lack of evidence for increased perceptions of fertility on the "red planet"
}

\author{
Richard D. Morey ${ }^{\mathrm{a}}$ and Malte Elson ${ }^{\mathrm{b}}$ \\ ${ }^{\text {a }}$ Cardiff University; ${ }^{\mathrm{b}}$ Ruhr University Bochum \\ Submitted on April 1, 2017
}

Throughout recorded history, fertility has been connected to both agriculture and human sexual reproduction. The two meanings often share representations or symbols across cultures, e.g. through depictions of Earth as a fertile woman or "mother". It is known that, to increase their reproductive success, human females signal their fertility to conspecifics with the color red. Given the strong link between human fertility to agricultural fertility, it stands to reason that humans should similarly perceive the soil on Mars - often referred to as "the red planet" - to be more fertile. We report here the results of an ambitious experiment that provides a compelling null effect in favor of this hypothesis. We discuss this observation and provide guidance for future planetary priming research.

fertility | red | plants | Mars | Earth | planetary priming

$\mathbf{T}$ he development of agriculture is often identified as the single most important cultural achievement by humans $(1,2)$. Agriculture heralded a number of changes in human society that allowed the growth of cities and nations, enabled humans to shape Earth environments to their needs and wants, and served as catalyst for major technological advances. The recent achievement of human spaceflight may prove to be another key moment in human history $(3,4)$. If humans are to successfully colonize other planets, agriculture will be crucial in shaping those planets to human needs. Understanding human perceptions of fertility will therefore be a life or death matter in the age of space colonization.

Human conceptions* of agricultural fertility have long been tied to concepts of human fertility. The idea of the Earth as "mother" is a common theme in world religions; religious celebrations of fertility often join the two explicitly. In art, the Earth is often depicted as a fertile woman, and symbols of agricultural fertility, such as flowers, often stand in for human genitalia (for further discussion, see (5)). These same symbols are exchanged in human courtship rituals. It is therefore likely that the cognitive connection between agricultural fertility and human fertility has deep evolutionary roots, perhaps reaching as far back as the development of sexual reproduction in single-celled organisms.

It has been shown using several methods $(6,7)$ that human females signal their fertility to conspecifics with the color red: for example, red lipstick, nail polish, clothing or even the face itself (8). Given that - from an evolutionary perspective human fertility and agricultural fertility are strongly linked, both in ultimate cause (the development of sexual reproduction in a common unicellular ancestor) and cognition, it stands to reason that planets signal their fertility in a similar way to humans; or, to be more precise, humans should perceive the soil on red planets to be more fertile.

*We note that even the word "conception" is, itself, related to sexual reproduction. This supports the idea that all human thought is connected through a unified notion of "fertility".

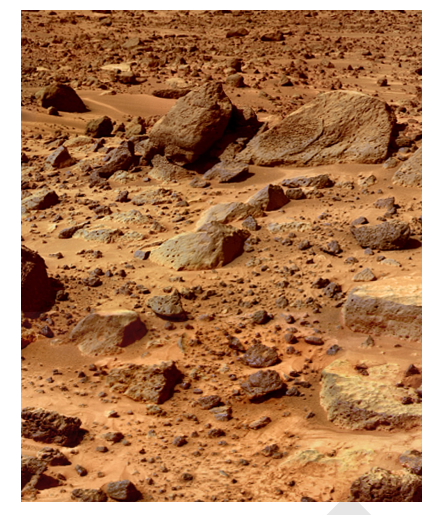

Fig. 1. The surface of Mars. (credit: NASA/JPL)

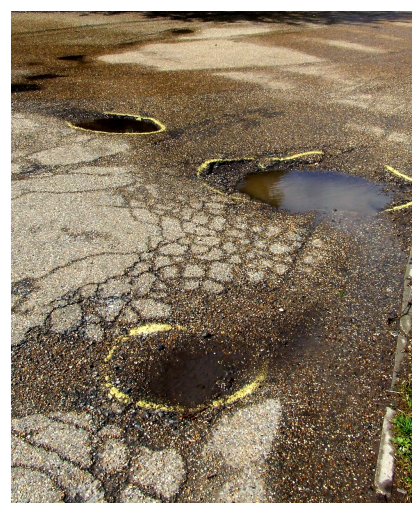

Fig. 2. The surface of Earth. (credit: Dave Bonta, CC-BY-SA 2.0)

We report here the results of an ambitious experiment to test the hypothesis that participants on Mars - often referred to as "the red planet" — perceive its surface (Fig. 1) as being more fertile than participants on Earth perceive its surface (Fig. 2). In order to test our hypothesis, we noted that animals and seed-bearing plants co-evolved: many plants bear fruit that is attractive to animals. Animals discard parts of fruits that are unpalatable, inedible, or undigestible, spreading the seeds to new areas $(9,10)$. We exploited this fact by defining a fertility perception ratio $(F P R)$ as the number of fruits a participant discarded on the ground (a "fertile" act, because the seeds may take root) divided by the number of fruits discarded in a waste receptacle (an infertile act, because these seeds will die). At the end of each day in the 14-day experimental period, participants $(N=0)$ were asked how many fruits they had discarded in each way. Participants were then categorized as either "high-fertility" actors $(F P R>1.53)$ or "low-fertility" actors $(F P R \leq 1.53)$.

\section{Significance Statement}

Like clothing, planets are different colors: Mars is red, while much of Earth is concrete-grey. Previous research has shown that humans have evolved so that wearing red is a signal of fertility. It is possible that we associate the color red with fertility because plants have domesticated us to prefer red planets; ultimately, they would like us to take them from Earth to Mars and beyond.

The authors each wasted equal time drafting this article.

Both authors declare that they live and work on Earth, which competes with Mars for inhabitants and research funding.

${ }^{1}$ E-mail: richarddmorey@gmail.com 
Table 1. Results of the experiment. Perceptions of fertility and planet were not significantly related $(p=1.00)$.

\begin{tabular}{lrr} 
& High Fertility $(F P R>1.53)$ & Low Fertility $(F P R<1.53)$ \\
\hline Earth & 0 & 0 \\
Mars & 0 & 0 \\
\hline
\end{tabular}

Participants completed the Attitudes towards Mars (ATOM) and the Obsession with Red Planets (ORP) scales (see supplemental information); participants scoring higher than 5.4 on either scale were eliminated from consideration. The data addressing the key question of interest are shown in the Table 1. The association between perceptions of fertility and the planet on which the participant was located was not significant at typical criteria $\left(p=1.00, \mathrm{CI}_{95 \%}\right.$ (tetrachoric $\left.\left.\rho\right): \emptyset\right)$. We take this as clear evidence that participants on Mars do not view the red soil on Mars as more fertile than participants on Earth view Terran soil.

\section{Discussion}

Given the strength of the evidence in the literature linking fertility and color, we were quite surprised by this negative result. We consider - and ultimately reject - several possible explanations: confusion with the masculinity of the Mars, confounds due to the lack of random assignment, low power, or lack of a true effect.

Cross-cultural effects. A reviewer helpfully noted that in Western mythology, the planet Mars is a masculine symbol: the Roman god of war. In fact, across many cultures Mars is identified as male. This raises the possibility that our lack of effect was due to participants not viewing Mars as fertile at all. Although we admit the possibility of cross-cultural effects in perceptions of planetary fertility, there were, in fact, no participants in our sample from cultures that view Mars as male. We can therefore rule out cross-cultural effects as an explanation for our null findings.

Random sampling. We did not randomly assign participants to the two conditions, which opens up the possibility of confounds due to, for example, self-selection into planetary condition. We considered the problem carefully and searched the statistical literature, but found no discussion of how to randomize no participants. Upon reflection, we considered random assignment superfluous, due to the fact that there is only $\left(\begin{array}{c}0+2-1 \\ 0\end{array}\right)=1$ way to assign the $N=0$ participants to the two conditions (that is, no participant in any condition). Any assignment is therefore equivalent to randomized assignment, because we would have obtained the same assignment either way. Nevertheless, we checked for significant differences in demographics between the groups. No significant differences were found for any demographic question (all $p \mathrm{~s}=1$ ). We therefore consider it unlikely that the lack of random assignment can explain our null effect.

Small sample size. A commenter on a previous version of this manuscript noted that the sample size in our study is low. After a thorough search of the literature, we admit that our sample size of $N=0$ may seem somewhat low compared to the standards in psychological research fields. However, our sample size is not atypical of studies of interplanetary differences in perceptions of fertility: a comprehensive search of the relevant literature revealed no such studies with a larger sample size.

It might be argued that standards for statistical power are changing in psychological science, and so we should have collected more participants. We caution, though, against thinking of sample size in a "one-size-fits-all" fashion and the fetishization of large samples (11). Here, we provide three major arguments why for many research questions - perhaps even most research questions in the psychological literature $N=0$ represents the best choice.

Efficiency. All research has resource constraints; statistical power must be weighed against the importance of the research in question and the costs. The questions address here are undoubtedly important, but they are also expensive. Bacchetti (12) has pointed out - and we agree - that statistical power must not trump cost-efficiency and feasibility; indeed, increasing our sample by just one further participant would have cost hundreds of billions of Euro. Moreover, with the small sample sizes we obtained, we could run many exploratory studies; at higher sample sizes, exploratory studies would be impossible.

Ethics. All research operates within legal and ethical boundaries. Researchers must ensure that one's design does not needlessly place participants at risk. Indeed, our institutional review board initially rejected the research proposal because we did not make sufficiently clear that the number of human participants sent to Mars would be indistinguishable from zero. Given the importance of the research question for humanity, however, not conducting the study at all would have equally violated our own ethical principles. Consequently, fixing the number of participants at 0 was the only morally defensible option. Furthermore, our local IRB immediately gave us a blanket approval for all future studies with $N=0$ subjects, facilitating our research by saving time and paperwork.

"Accepting" the null. The burden for a study whose null results would falsify a hypothesis is quite high given that the absence of evidence for an effect is not the same as evidence of its absence. Using $N=0$ subjects largely resolves this philosophical dilemma: it allows the possibility of truly observing no effect. In our view, the evidence of absence we provide is the strongest possible. This is particularly true for NeymanPearson frequentist inference primarily concerned with error control because it is literally impossible to err. Where there's no measurement, there is no effect, and thus no error.

Lack of a true effect. Given that we have shown definitively that there is no "Red Planet" effect on perceptions of fertility, one might be tempted then to infer that planets do not appear to signal their fertility via their color. This would be a mistake. In any study, the statistical results are only part of the story. When deciding the evidential impact of an experiment, one must assess the totality of the evidence. The statistical results do not take into account the incredibly low probability that anyone, anywhere, would ever want to test the hypothesis we suggest. The low a priori probability of generating our hypothesis in the first place suggests that - perhaps - there is a deeper reason behind its generation. To understand what this deeper reason might be, we consider again the domestication of plants at the dawn of human civilization.

Although it is often thought that humans domesticated plants, this perspective is inappropriately anthropocentric. In 
fact, it is possible to view the development of agriculture as plants having domesticated homo sapiens. ${ }^{\dagger}$ Humans have, in a real sense, been selected to fill an ecological niche and spread plants across the Earth. When we look up at the stars and make our plans for terraforming other planets, we are acting out hundreds of thousands of years of evolutionary manipulation of the human species by plants. Our desire to perform this study must be understood as evidence that plants would have us scatter them across the emptiness of interplanetary space. Like a bird dropping a seed far from its parent, we, too, will eventually take the plants where they cannot go alone.

Thus, on balance, our results suggest that further research for this important question is needed. We believe the $h u$ man domestication by plants perspective will prove fruitful in framing future planetary fertility research. That we are even suggesting such an implausible hypothesis - along with the fact that we are suggesting it at precisely the moment when interplanetary human spaceflight has become possible - is what one would predict under the theory that humans have been domesticated by plants. Our null finding and subsequent call for further research should call the hypothesis into doubt; if anything, the opposite is true.

\section{Materials and Methods}

The Attitudes towards Mars (ATOM) and the Obsession with Red Planets (ORP) scales may be found in the supplemental information to this article.

Computing $p$ values. There is surprisingly little statistical literature describing how to compute $p$ values for $N=0$ designs. After several

1. Trotter WH (1998) Microsoft Age of Empires: Inside Moves ed. Kent P. (Microsoft Press,U.S.), 2nd revised edition edition edition.

2. Ensemble Studios (1997) Age of Empires.

3. Brady Games (2010) Civilization V Official Strategy Guide. (Brady Games, Indianapolis, IN).

4. Firaxis Games (2013) Civilization V: Brave New World.

5. Jung CG, ed. (1964) Man and his symbols. (Anchor Press, New York).

6. Beall AT, Tracy JL (2013) Women are more likely to wear red or pink at peak fertility. Psychological Science 24:1837-1841

7. Tracy JL, Beall AT (2014) The impact of weather on women's tendency to wear red or pink when at high risk for conception. PLOS ONE 9(2):e88852.

8. Kret ME, Tomonaga M (2016) Getting to the Bottom of Face Processing. Species-Specific Inversion Effects for Faces and Behinds in Humans and Chimpanzees (Pan Troglodytes). PLOS ONE 11(11):e0165357.

9. van der Pijl L (1982) Principles of Dispersal in Higher Plants. (Springer-Verlag, Berlin).

10. Wall SBV (2010) How plants manipulate the scatter-hoarding behaviour of seed-dispersing animals. Philosophical Transactions of the Royal Society of London B: Biological Sciences failed attempts at solving the problem using Monte Carlo simulation, we discovered an analytical solution. If we regard the $p$ value as the probability of obtaining more extreme evidence under the null hypothesis, we then note that it is impossible to have obtained less evidence than we did. Therefore, $p=1$.

Our method of computing $p$ values has a Type I error rate of exactly 0 , which is somewhat conservative. As noted in the text with $N=0$, by definition there is no effect and hence the Type II error rate is not an issue. We have, apparently, discovered a perfect statistical technique (a manuscript detailing this new method is forthcoming in Frontiers in Statistical Inference).

Computing confidence intervals. In order to compute confidence intervals, we adapted a method suggested by the statistician Basu (14, Example 6). We took as our parameter of interest the tetrachoric correlation coefficient for $2 \times 2$ contingency tables. Knowing that for $N=0$ the true tetrachoric $\rho$ is undefined, we defined a confidence procedure as follows:

1. Roll a twenty-sided die (15);

2. If the die shows " 20 ", the confidence interval is $[-1,1]$;

3. Otherwise, the confidence interval is the null set $\emptyset$.

For $N=0$, the procedure is guaranteed to have the proper $95 \%$ coverage. For $N>0$, the outcomes can be reversed to obtain the expected coverage.

Data availability. The data from this study are available here upon request. Send all requests via post to Lamby Way Waste Depot, Cardiff CF3 2HP, United Kingdom.

ACKNOWLEDGMENTS. We would like to thank Sol's protoplanetary accretion disk for making this research possible.

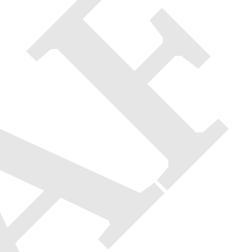

365(1542):989-997.

11. Baumeister RF (2016) Charting the future of social psychology on stormy seas: Winners, losers, and recommendations. Journal of Experimental Social Psychology 66:153-158.

12. Bacchetti $P$ (2013) Small sample size is not the real problem. Nature Reviews Neuroscience 14(8):585-585.

13. Calvo P, Sahi V, Trewavas A (2017) Could Plants Be Sentient? bioRxiv p. 121731

14. Basu D (1981) On ancillary statistics, pivotal quantities, and confidence statements in Topics in Applied Statistics, eds. Chaubey YP, Dwivedi TD. (Concordia University, Montreal), pp. $1-29$.

15. (2014) Dungeons \& Dragons Player's Handbook. (Wizards of the Coast, Renton, WA).

${ }^{\dagger}$ It has recently been suggested that plants may be sentient (13). We would not be surprised if plants were sentient; in fact, the government of the United States appears to be controlled by vegetables. It only in this context that one can understand the urgency with which the current administration pursues a manned Mars mission. 


\section{Attitudes Towards Mars Scale (ATOM)}

\begin{tabular}{|c|c|c|c|c|c|}
\hline & $\begin{array}{c}1 \\
\text { Even less } \\
\text { neutral in the } \\
\text { "No" direction }\end{array}$ & $\begin{array}{c}2 \\
\text { Less neutral } \\
\text { in the } \\
\text { "No" direction }\end{array}$ & $\begin{array}{c}3 \\
\text { Neutral }\end{array}$ & $\begin{array}{c}4 \\
\text { Less neutral } \\
\text { in the } \\
\text { "Yes" direction }\end{array}$ & $\begin{array}{c}5 \\
\text { Even less } \\
\text { neutral in the } \\
\text { "Yes" direction }\end{array}$ \\
\hline Mars is very important to me personally & 0 & 0 & 0 & 0 & 0 \\
\hline I like learning more about Mars & 0 & 0 & 0 & 0 & 0 \\
\hline I frequently recommend Mars to others & 0 & 0 & 0 & 0 & 0 \\
\hline Of all red planets in our solar system, Mars is easily the best & 0 & 0 & 0 & 0 & 0 \\
\hline $\begin{array}{l}\text { I have to restrain myself when interacting with Jovian } \\
\text { enthusiasts }\end{array}$ & 0 & 0 & 0 & 0 & 0 \\
\hline $\begin{array}{l}\text { I always speak favorably of Mars regardless of what Minerva } \\
\text { thinks }\end{array}$ & 0 & 0 & 0 & 0 & 0 \\
\hline I find the thought of Snickers repulsive & 0 & 0 & 0 & 0 & 0 \\
\hline I like to think of Mars as my home & 0 & 0 & 0 & 0 & 0 \\
\hline I'm a "down-to-Earth" kind of person [reverse-coded] & 0 & 0 & 0 & 0 & 0 \\
\hline
\end{tabular}




\section{“Obsession with Red Planets" scale (ORP)}

The "Obsession with Red Planets" scale (ORP) is copyrighted by Richard D. Morey, (C) 2017. Any use of the material in this document other than reading and promptly forgetting it without written permission of the copyright holder is expressly prohibited. Violations are actionable under APA Standard 9.11 and subject to punishment by waterboarding.

Items begin on the next page. By viewing these items you agree to the terms of use. 
Please indicate the extent to which you agree with the following statements. Use the 5 point scale indicated below. Circle the number that corresponds to your level of agreement.

\begin{tabular}{|l|l|l|l|l|}
\hline 1 & \multicolumn{1}{|c|}{2} & \multicolumn{1}{|c|}{3} & \multicolumn{1}{c|}{4} & \multicolumn{1}{c|}{5} \\
\hline Disagree & $\begin{array}{l}\text { Agree more than } \\
\text { would be implied } \\
\text { had I selected the } \\
\text { option } \\
\text { immediately to } \\
\text { the left of this } \\
\text { one }\end{array}$ & $\begin{array}{l}\text { Agree more than } \\
\text { would be implied } \\
\text { had I selected the } \\
\text { option } \\
\text { immediately to } \\
\text { the left of this } \\
\text { one }\end{array}$ & $\begin{array}{l}\text { Agree more than } \\
\text { would be implied } \\
\text { had I selected the } \\
\text { option } \\
\text { immediately to } \\
\text { the left of this } \\
\text { one }\end{array}$ & $\begin{array}{l}\text { Agree more than } \\
\text { would be implied } \\
\text { had I selected the } \\
\text { option } \\
\text { immediately to } \\
\text { the left of this } \\
\text { one }\end{array}$ \\
\hline
\end{tabular}

1. I see red when I think about Mars
1
2
3
4
5

2. I often dream that I am a functioning NASA Mars rover
1
2
3
4
5

3. I frequently check the Kepler project's press releases for newly-discovered red planets
1
2
3
4
5

4. If I could, I would make all the planets red
1
2
3
4

5. I believe that there is a scientific conspiracy to hide the canals on Mars

1

2

3

4

(continued on the next page) 
6. The red blood that flows through my veins yearns only for a red planet to call home. The day I settle that planet, I will destroy those that tried to smother my dream. Though their screams are muffled by the thin atmosphere, I hear them in my mind's ear. Crimson flames will leave only cinders on the once-green Earth

1 2

7. I wish I were a Mars One $\mathrm{TM}^{\mathrm{TM}}$ astronaut 2 3 4

8. I sometimes wake up on Mars

1 2 3

9. My friends tell me I talk about Mars too much

1 2 3

10. The statements above have aroused me 